Kuenzle, C. C. (1970b) Biochem. J. 119, 411-435

Kuenzle, C. C. (1973) in Metabolic Conjugation and Metabolic Hydrolysis (Fishman, W. H., ed.), vol. 3, Academic Press, New York, in the press
Kuenzle, C. C., Weibel, M. H., Pelloni, R. R. \& Hemmerich, P. (1973), Biochem. J. 133, 364-368

von Dobeneck, H. \& Brunner, E. (1965) Hoppe-Seyler's Z. Physiol. Chem. 341, 157-166

\title{
APPENDIX
}

\section{Structure and Conformation of Bilirubin}

\section{OPPOSING VIEWS THAT INVOKE TAUTOMERIC EQUILIBRIA, HYDROGEN BONDING AND A BETAINE MAY BE RECONCILED BY A SINGLE RESONANCE HYBRID}

\author{
By Clive C. KUENZLE,* MATTHIAS H. WEIBEL,* RENATO R. PELLONI* and \\ PETER HEMMERICH $\dagger$ \\ * Department of Pharmacology and Biochemistry, School of Veterinary Medicine, \\ University of Zürich, $\mathrm{CH}-8057$ Zürich, Switzerland and \\ $\dagger$ Department of Biology, University of Konstanz, D-7750 Konstanz, Germany
}

\begin{abstract}
A novel conformational structure of bilirubin is presented which obtains maximum stabilization through a system of four intramolecular hydrogen bonds. Two hydrogen bonds link oxygen and nitrogen atoms of each end ring to the contralateral carboxyl group. The proposed structure can explain a variety of uncommon features of bilirubin, and reconciles many seemingly contradictory hypotheses by accommodating them in individual structures which are mesomeric forms of one resonance hybrid. In the light of this newly conceived structure the following characteristics of bilirubin are re-evaluated: the stability of the compound, its reaction with diazomethane, the conformational behaviour of its dimethyl ester, its spectral properties, the chirality of the compound when complexed to serum albumin, and the structure of its metal chelates.
\end{abstract}

The basic structure of bilirubin was elucidated by Fischer's group (Fischer et al., 1941; Fischer \& Plieninger, 1942). Nevertheless, some anomalous properties of the substance have stimulated further research. In the first instance, Fischer's original proposal that bilirubin exists as the bis-lactim had to be modified on the strength of evidence gained from other ' $\alpha$-hydroxypyrroles' (Plieninger \& Decker, 1956; Plieninger et al., 1962) and from spectra of bilirubin and its derivatives (Gray et al., 1961; von Dobeneck \& Brunner, 1965; Kuenzle, 1970a). Thus today it is common usage to represent bilirubin with the two outer rings in the lactam configuration. An exception to this is a recent claim for the prevalence of the bis-lactim form (Nichol \& Morell, 1969), but this inference was based on misinterpreting the n.m.r. spectrum of bilirubin (Kuenzle, 1970a). However, present-day spectroscopy cannot exclude the occurrence of very small quantities of a bis-lactim tautomer at equilibrium. This has been invoked in explanation of mono- and di-methoxy derivatives forming when bilirubin is esterified with diazomethane (Kuenzle, 1970a).

Prompted by the experience that bilirubin is much less prone to oxidation than both its anion and its naturally occurring acyl glycosides, Fog and coworkers (Fog \& Bugge-Asperheim, 1964; Fog \& Jellum, 1963) have suggested that this may be due to the stabilizing effect of intramolecular hydrogen bonds existing between the carboxylic acid groups and the pyrrole nitrogen atoms of the inner rings. Other types of hydrogen bonds have been implicated by Brodersen et al. (1967), Nichol \& Morell (1969), Kuenzle (1970a) and Hutchinson et al. (1971). To support the various hypotheses, the unusually low i.r. frequencies associated with the carboxyl $\left(1680 \mathrm{~cm}^{-1}\right)$ and amide $\left(1645 \mathrm{~cm}^{-1}\right)$ functions have been invoked (Fog \& Jellum, 1963; Brodersen et al., 1967; Nichol \& Morell, 1969; Hutchinson et al., 1971). However, neither of the proposed structures has proved completely convincing, either because in devising the structure steric requirements have been 
neglected, or because no significant stabilization has been apparent from the proposed hydrogen bonding.

Another peculiarity of bilirubin is its electronic spectrum. Model dipyrromethenes of the 'neo'-type, i.e. those containing a pyrrole and a pyrrolenone ring interlinked by $\mathrm{a}-\mathrm{CH}=$ bridge, all absorb at around $400 \mathrm{~nm}$ and this maximum is shifted to approx. $450 \mathrm{~nm}$ on acidification (von Dobeneck \& Brunner, 1965). In contrast, bilirubin peak absorption is at $453 \mathrm{~nm}$ and is unchanged in acid. On the other hand, strongly alkaline solvents convert bilirubin into its anion, which absorbs at $424 \mathrm{~nm}$. This has been inferred to indicate that bilirubin exists as a betaine (von Dobeneck \& Brunner, 1965). Support for this hypothesis has been adduced by i.r. spectroscopy. The N-H stretching frequency resembles that for the hydrobromide of a dipyrromethene and other quaternary compounds $\left(\mathrm{N}-\mathrm{H}, 3400-3425 \mathrm{~cm}^{-1}\right)$, but differs from that of non-quaternary dipyrromethenes $(\mathrm{N}-\mathrm{H}$, approx. $3333 \mathrm{~cm}^{-1}$ ) (von Dobeneck \& Brunner, 1965).

The following arguments support a novel bilirubin structure, which is not only compatible with the many uncommon features of the pigment but which also constitutes a resonance hybrid that accommodates all previously proposed structures in mesomeric forms (Fig. 1).

\section{Stability of bilirubin}

The structure proposed for bilirubin gains maximal stabilization from four hydrogen bonds. It is easy to see how alkali will destabilize the system by abstracting the hydrogen atoms involved in the outer hydrogen bonds, which will thus be abolished. Bilirubin conjugates of bile and jaundiced serum occur with esterified propionic acid groups (Kuenzle, 1970b; Fevery et al., 1971; Compernolle et al., 1971). Thus, as with bilirubin dimethyl ester (Scheme 1, VII), stabilization by the proposed hydrogen bonding is largely lost. It is possible also that inhibition of hydrogen-bond formation occurs as a consequence of solvation by water molecules.

\section{Mechanism of the reaction of bilirubin with diazo- methane}

The ambiguity that arises from the proposed hydrogen bonds with respect to assigning the outer hydrogen atoms to either the carboxyl or the iminol groups is the key to understanding the reaction mechanism of methylation of bilirubin with diazomethane. This reaction not only yields the expected dimethyl ester but also produces some monomethoxy- and dimethoxy-bilirubin dimethyl ester. In the latter compounds the pyrrolenone rings are transformed to feature a lactim ether configuration (Kuenzle et al., 1973). Although this product distribution might also
(I)

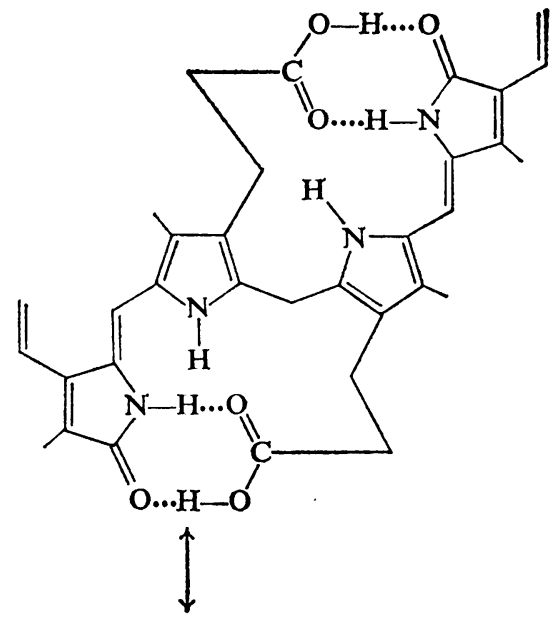

(II)<smiles>C=CC1=C(C)C(O[C@@H](C)OC)NC1=CC</smiles>

(III)<smiles>[3H][3H]</smiles>

(IV)<smiles>C=CC1=C(C)C(=O)N([C@H](O)OC(C)=O)/C1=C\C</smiles><smiles>[3H][3H]</smiles><smiles></smiles>

Fig. 1. Mesomeric structures of bilirubin

Note the four intramolecular hydrogen bonds and the delocalized positive charge on the chromophores. Only the relevant structures are shown in formulae (II)-(IV).

be referred to the possible occurrence of an amidoiminol tautomerism, the presence of such an equilibrium would not explain the fact that the methoxy

Vol. 133 
derivatives cannot be prepared when bilirubin dimethyl ester replaces bilirubin as the starting material (Kuenzle, 1970a). The observations, however, are perfectly compatible with the reaction sequence of
Scheme 1, which follows divergent routes depending on to which side the methyl group is attracted after it has been introduced by the diazomethane reaction with a hydrogen of the outer hydrogen bond. The
(VI)<smiles>C=CC1=C(C)C(=O)N([C@H]2O[C@@H](C)O[C@@H]([N+]#N)[C@H]2[N+]#N)C1=O</smiles>

(VII)<smiles>C=CC1=C(C)C(=O)N([C@H](C)OC(C)OC)/C1=C/C</smiles><smiles>C/C=C\CN1C(=O)O[C@H]2OC(C)O[C@@H]21</smiles><smiles>C=CC1=C(C)C(=O)N(C[C@H]2CC[C@H](C)O2)C1=O</smiles><smiles>C=CC1=C(C)C(OC)=N/C1=C\C</smiles>

(X)

Scheme 1. Molecular mechanism of the reaction of bilirubin with diazomethane

The outer hydrogen-bonding hydrogen atoms of the bilirubin sustain nucleophilic abstraction by the polarized reagent $(\mathrm{V})$. Electrophilic attack by the protonated methylene group of the diazomethane ensues either at the carboxyl group (VI) or, after mesomeric transfer of the anionic charge $(\curvearrowright)$, at the pyrrolenone carbonyl group (VIII). Bilirubin dimethyl ester (VII) or mono- and di-methoxy bilirubin (IX) are formed respectively and the methoxy bilirubins are then further methylated to dimethyl esters (X). 


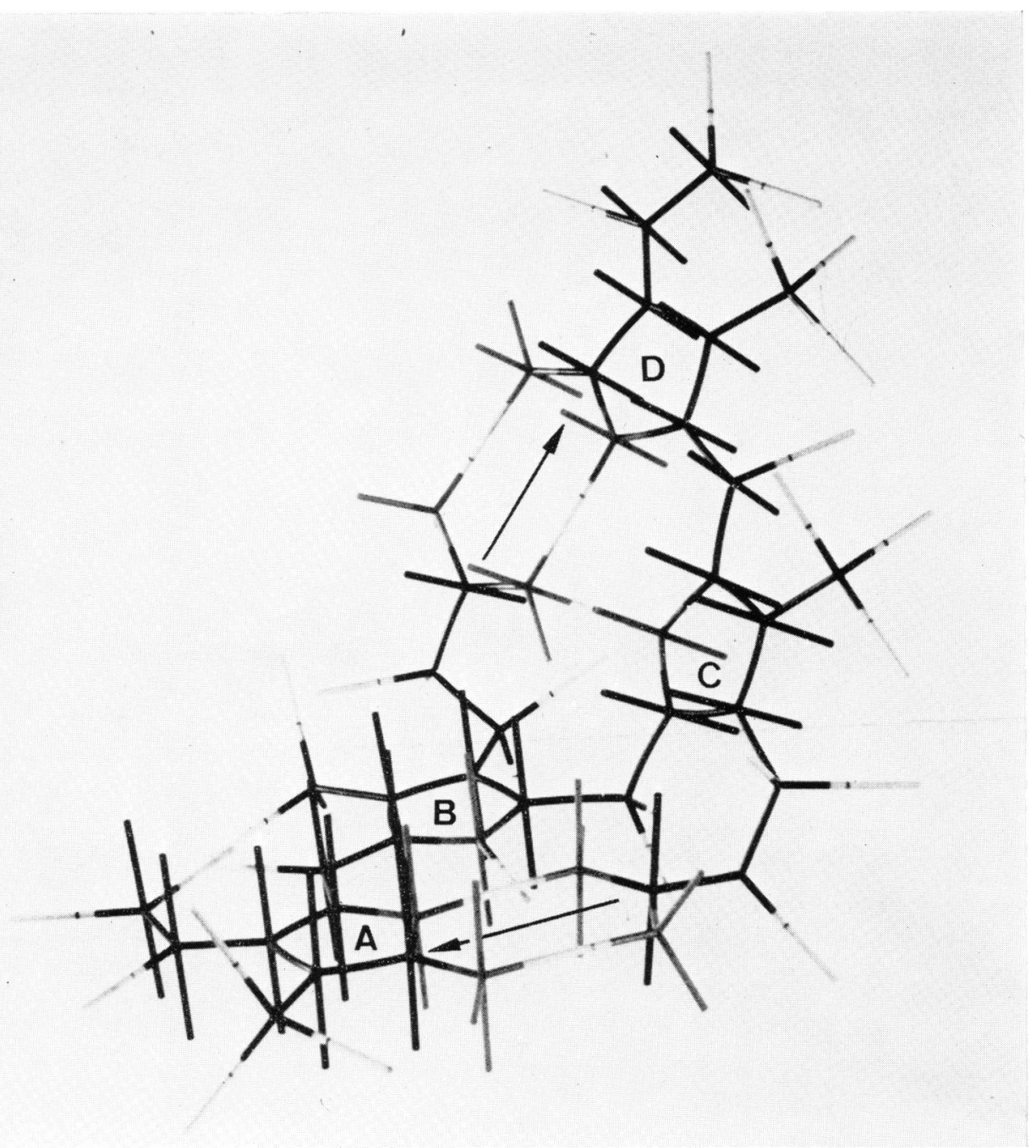

\section{EXPLANATION OF PLATE I}

Molecular model of bilirubin demonstrating one of the two enantiomeric conformations that ensue from intramolecular hydrogen bonding

Note the two molecular planes separated by the central methylene bridge. Conversion into the enantiomeric conformation requires breakage of all four hydrogen bonds, rotation about the central methylene bridge resulting in a downward orientation of the right-hand side half-molecule and re-formation of the hydrogen bonds. Hydrogen atoms are shown in light grey, carbon atoms in black, oxygen atoms and nitrogen atoms in dark grey. The bars that are not directly involved in bond formation either represent $\pi$ orbitals or, in the case of oxygen, orbitals that accommodate free electron pairs. The arrows mark the two hydrogen-bond systems and are interposed between the inner and the outer hydrogen bonds in a parallel arrangement; their direction is from the carboxyl groups to the pyrrolenone rings. Rings are labelled A-D. 
methyl group is thus either accommodated on the carboxyl site yielding bilirubin dimethyl ester (VII) directly or is diverted to the pyrrolenone oxygen, in which case an intermediate mono- or di-methoxybilirubin (IX) reacts with a further molecule of diazomethane to afford the final product of monoor di-methoxybilirubin dimethyl ester (X). This sequence of reactions is dependent on the portrayed arrangement of the hydrogen bonds and thus supports the overall structure proposed for bilirubin.

\section{Restricted rotation about the central methylene bridge in bilirubin dimethyl ester}

Bilirubin dimethyl ester dissolved in non-polar solvents (chloroform and carbon tetrachloride) has been shown by n.m.r. spectroscopy to exist as two distinct molecular species (Kuenzle, 1970a). This behaviour can now be explained as resulting from a hydrogen-bonded structure analogous to the one proposed for bilirubin (Fig. 1, I) except for the absence of the two outer hydrogen bonds (Scheme 1, VII). However, it is suggested that the two remaining inner bonds that link the ester carbonyl oxygen atoms to the amide hydrogen atoms provide sufficient stability to restrict rotation about the central methylene bridge. This partial 'freezing' of the system allows the hydrogen-bonded conformation to be recognized by n.m.r. spectroscopy as one of two molecular species, whereas a family of freely rotating, non-bonded conformations accounts for the other. This interpretation is supported by the recent finding (Kuenzle et al., 1973) that under identical conditions dimethoxybilirubin dimethyl ester registers as a single molecular species, in agreement with the lack of the structural features necessary for hydrogen bonding (Scheme 1, X).

\section{Spectral properties of bilirubin and the question of a betaine structure}

Compelling evidence for a betaine structure of bilirubin has been adduced from its electronic and i.r. spectra (von Dobeneck \& Brunner, 1965). The present structural proposal is in agreement with this hypothesis since it features an extensively delocalized positive charge spread over the entire chromophore (see for example the mesomeric structures III and IV in Fig. 1). This formulation supersedes the objections raised previously against a betaine in the classical sense (Kuenzle, 1970a) with the molecular conformation being totally in accord with all spectroscopic data so far obtained.

\section{Chirality of bilirubin}

Bilirubin is not evidently dis-symmetric when viewed in a conventional line representation. How- ever, Blauer \& King (1970) have pointed out that the molecule can assume 'inherently dis-symmetric' conformations as defined by Moscowitz et al. (1964). In fact, although no optical activity is observed with bilirubin in solution, the compound exhibits one of the largest Cotton effects reported in the visible region when complexed with bovine serum albumin (Blauer \& King, 1970). The rotatory properties of the complex are somewhat different with human serum albumin, particularly with reference to a characteristic inversion of the sign of the Cotton effect on changing the $\mathrm{pH}$ of the solution or on raising its ionic strength (Blauer et al., 1970, 1971, 1972; Blauer \& Harmatz, 1972). This inversion certainly reflects an altered bilirubin conformation, and more specifically, the symmetry of the inverted circular-dichroic spectra suggests the interconversion of an enantiomeric pair of conformations (Blauer et al., 1972; Blauer \& Harmatz, 1972). Indeed, Blauer and co-workers (Blauer \& King, 1970; Blauer et al., 1972) have proposed three sets of dis-symmetric bilirubin conformations that would conform to such an interpretation. Similarly, our own hydrogen-bonded structure (Fig. 1) is in accord with enantiomeric interconversion and this can be simulated with the use of molecular models. Thus breakage of all four hydrogen bonds followed by rotation about the central methylene bridge and re-formation of the hydrogen bonds leads to two chiral conformations that share a common mirror-plane defined by the carbon skeleton of the one chromophore visualized as remaining in a fixed position (Plate 1). On the basis of this observation we support the proposal of Blauer and his colleagues (Blauer et al., 1972; Blauer \& Harmatz, 1972) that the reported inversion of the Cotton effect may reflect a solvent-dependent refolding of the albumin chain allowing the bilirubin receptor to assume two different conformational states which would be characterized by their preferential avidity for either one of the two enantiomeric bilirubin conformations.

\section{Metal chelates of bilirubin}

Bilirubin forms complexes with transition metals and lanthanides (Ó Carra, 1962; Fog \& Bugge-Asperheim, 1964; Van Roy \& Heirwegh, 1965; Nichol \& Morell, 1969; Velapoldi \& Menis, 1971). Since their original description these complexes have been tacitly assumed to have structures analogous to those of the metalloporphyrins, in which a single metal ion is surrounded by four nitrogen atoms grouped in a quasi-square-planar arrangement. However, steric considerations must raise serious doubts as to the stability of such a configuration in complexes of bilirubin. With this in mind, we have recently proposed a chelate structure in which the salient features contributing to the stability of the uncomplexed bilirubin are preserved (although in a modified form) and 
the steric requirements are essentially fulfilled (see Fig. 1 in Kuenzle et al., 1972). Thus, whereas the outer hydrogen bonds of bilirubin continue to link the carboxyl groups to the pyrrolenone oxygen atoms, the inner hydrogen bridges are replaced by a system of nitrogen-metal and oxygen-metal bonds providing trigonal co-ordination for one metal ion in each of the two molecular planes. The complexing of 2 metal equivalents/mol of bilirubin has been substantiated by spectrophotometric titration with $\mathrm{Sm}^{3+}$ and by the markedly differing stability constants for the incorporation of the first and second metal ion (Kuenzle et al., 1972). It has been suggested that this arises from steric interaction between additional ligands extending upwards from the molecular planes, thus affording evidence for a co-ordination number greater than three, but the exact number of ligands and their correct orientations are not known as yet. In spite of this shortcoming the experimental evidence reported above lends strength to the contention that not only are the essential features of the complex correctly represented but so also is the hydrogenbonded structure proposed for bilirubin.

\section{References}

Blauer, G. \& Harmatz, D. (1972) Biochim. Biophys. Acta 278, 89-100

Blauer, G. \& King, T. E. (1970) J. Biol. Chem. 245, 372-381

Blauer, G., Harmatz, D. \& Naparstek, A. (1970) FEBS Lett. 9, 53-55

Blauer, G., Harmatz, D. \& Snir, J. (1971) Proc. Eur. Biophys. Congr., 1st, pp. 127-132

Blauer, G., Harmatz, D. \& Snir, J. (1972) Biochim. Biophys. Acta 278, 68-88
Brodersen, R., Flodgaard, H. \& Krogh Hansen, J. (1967) Acta Chem. Scand. 21, 2284-2285

Compernolle, F., van Hees, G. P., Fevery, J. \& Heirwegh, K. P. M. (1971) Biochem. J. 125, 811-819

Fevery, J., van Hees, G. P., Leroy, P., Compernolle, F. \& Heirweigh, K. P. M. (1971) Biochem. J. 125, 803-810

Fischer, H. \& Plieninger, H. (1942) Hoppe-Seyler's Z. Physiol. Chem. 274, 231-260

Fischer, H., Plieninger, H. \& Weissbarth, O. (1941) Hoppe-Seyler's Z. Physiol. Chem. 268, 197-226

Fog, J. \& Bugge-Asperheim, B. (1964) Nature (London) 203, 756-757

Fog, J. \& Jellum, E. (1963) Nature (London) 198, 88-89

Gray, C. H., Kulczycka, A. \& Nicholson, D. C. (1961) J. Chem. Soc. 2276-2285

Hutchinson, D. W., Johnson, B. \& Knell, A. J. (1971) Biochem. J. 123, 483-484

Kuenzle, C. C. (1970a) Biochem. J. 119, 395-409

Kuenzle, C. C. (1970b) Biochem. J. 119, 411-435

Kuenzle, C. C. Pelloni, R. R., Weibel, M. H. \& Hemmerich, P. (1972) Biochem. J. 130, 1147-1150

Kuenzle, C. C., Weibel, M. H. \& Pelloni, R. R. (1973) Biochem. J. 133, 357-364

Moscowitz, A., Krueger, W. C., Kay, I. T., Skewes, G. \& Bruckenstein, S. (1964) Proc. Natl. Acad. Sci. U.S. 52, 1190-1194

Nichol, A. W. \& Morell, D. B. (1969) Biochim. Biophys. Acta 177, 599-609

Ó Carra, P. (1962) Nature (London) 195, 899-900

Plieninger, H. \& Decker, M. (1956) Justus Liebigs Ann. Chem. 598, 198-207

Plieninger, H., Bauer, H. \& Katritzky, A. R. (1962) Justus Liebigs Ann. Chem. 654, 165-180

Van Roy, F. \& Heirwegh, K. (1965) Arch. Int. Physiol. Biochim. 73, 535-536

Velapoldi, R. A. \& Menis, O. (1971) Clin. Chem. 17, 11651170

von Dobeneck, H. \& Brunner, E. (1965) Hoppe-Seyler's Z. Physiol. Chem. 341, 157-166 